\title{
Primary Sjögren's syndrome initially presenting as submandibular mucosa-associated lymphoid tissue lymphoma: A case report
}

\author{
LIANG-YU CHEN ${ }^{1}$, MING-HSUI TSAI ${ }^{1,2}$, LI-TAI TSAI $^{1}$, HSIN-MAN LU $^{3}$ and CHIA-ING JAN ${ }^{4-6}$ \\ ${ }^{1}$ Department of Otolaryngology - Head and Neck Surgery, China Medical University and Hospital; \\ ${ }^{2}$ Graduate Institute of Clinical Medical Science, China Medical University, Taichung 40402; \\ ${ }^{3}$ Department of Psychology, Asia University, Taichung 41354; \\ ${ }^{4}$ Department of Pathology, China Medical University Hospital, Taichung 40402; \\ ${ }^{5}$ Department of Pathology, China Medical University, Beigang Hospital, Beigang, Yunlin 65152; \\ ${ }^{6}$ Department of Dentistry, National Yang-Ming University, Taipei 11221, Taiwan, R.O.C.
}

Received September 24, 2014; Accepted May 29, 2015

DOI: 10.3892/ol.2015.3980

\begin{abstract}
The present study reports the case of a 24-year-old female affected with primary Sjögren's syndrome (pSS), who presented with mucosa-associated lymphoid tissue (MALT) lymphoma of the submandibular gland. Reports of such cases, particularly in young patients, are very rare. The patient, who presented no oral or ocular symptoms prior to the development of the mass, underwent surgical ablation of the gland, and MALT lymphoma was diagnosed by histopathology. Since MALT lymphoma in the submandibular gland is rarely observed in otherwise healthy young females, a rheumatologist and an oncologist were consulted. Following a number of immunological tests, the results of the Schirmer's and Saxon tests were negative. However, the antinuclear antibody test revealed a speckled appearance, and there was also strong positivity for the serological markers of Sjögren's syndrome. Consequently, pSS was diagnosed, despite the fact that the patient did not fulfill all the diagnostic criteria for the disease. Therefore, MALT lymphoma in a single salivary gland should be used as a differential diagnosis for Sjögren's syndrome in young asymptomatic patients. Additionally, a multidisciplinary team is required for the treatment and management of these patients.
\end{abstract}

\section{Introduction}

Sjögren's syndrome is an autoimmune disease characterized by lymphocytic infiltration of the exocrine glands, mainly the salivary and lacrimal glands (1). This leads to a loss of

Correspondence to: Dr. Chia-Ing Jan, Department of Pathology, China Medical University Hospital, 2 Yu-Der Road, North District, Taichung 40402, Taiwan, R.O.C.

E-mail: janc1206@yahoo.com.tw

Key words: Sjögren's syndrome, mucosa associated lymphoid tissue lymphoma, submandibular swelling secretory function, and patients with Sjögren's syndrome commonly present with oral or ocular symptoms, such as xerostomia or xerophthalmia (2). Sjögren's syndrome most commonly affects females in their 50 s and 60 s (3). In a recent systematic review of 21 studies, the pooled incidence and prevalence of primary Sjogren's syndrome (pSS) was estimated at 60.82/100,000 persons (4), and the mortality ratio reported in another meta-analysis in patients with pSS was $682 / 7,888$ persons over a median average follow-up of 9 years, respectively (5). Whereas symptomatic and topical treatments are essential in most patients with limited glandular disease. For systemic complication of the disease, conventional immunomodulatory treatements such as hydroxychloroquine and azathioprine or the more specific target specific anti-B lymphocytic therapeutic agents such as interferon inhibition, interleukin- 6 or interleukin-2 inhibition, and inhibition of B-cell activating factor are now suggested possible treatments (6).

Mucosa-associated lymphoid tissue (MALT) lymphoma is defined as an extranodal presentation of a low-grade B-cell lymphoma. MALT lymphomas may also develop in the salivary glands, lungs, stomach or lacrimal glands, and are commonly associated with pre-existing autoimmune diseases or prolonged immune stimulation, such as long-standing Sjögren's syndrome or chronic Helicobacter pylori (H. pylori) infection, respectively (7). It is established that the development of MALT lymphoma in the salivary gland can occur in long-term autoimmune disease (7). The present report is a rare case of a young female affected with Sjögren's syndrome who presented with a single submandibular MALT lymphoma.

\section{Case report}

A 24-year-old Chinese female with no history of drug use, including tobacco, was admitted to China Medical University Hospital (Taichung, Taiwan) on June 2014 presenting with a non-tender left sub-mandibular mass, which started to grow 3 months prior to the consultation, with rapid enlargement in the third month. The patient was otherwise asymptomatic, with no clear signs of xerostomia or xerophthalmia, and had 

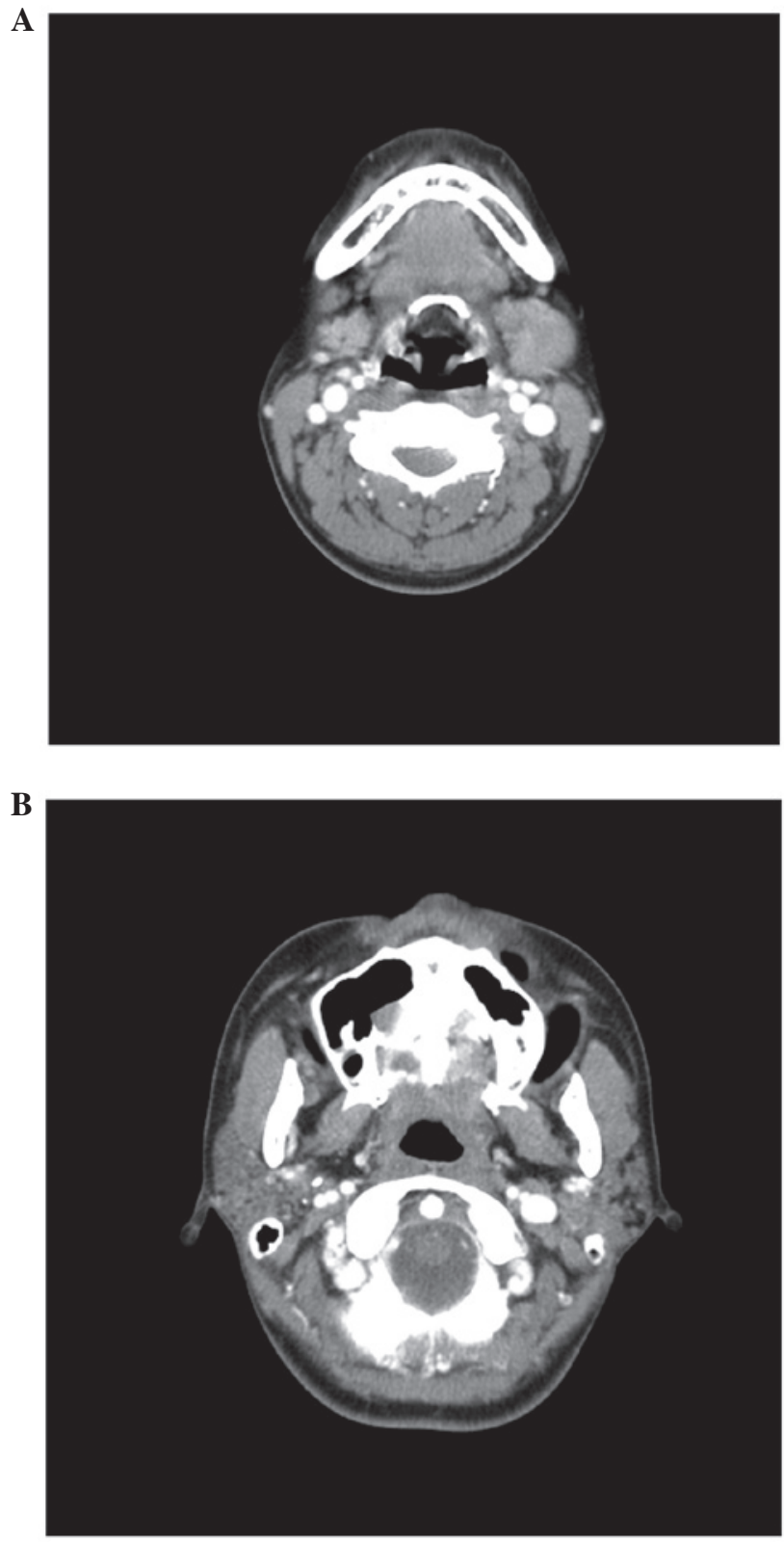

Figure 1. Enhanced axial computed tomography imaging. (A) A single enlarged submandibular gland. (B) Heterogeneous appearance and multiple microcystic changes in the bilateral parotid glands.

no history of malignancy, coagulation disorders, arthralgia or autoimmune disease.

Physical examination revealed a firm, movable mass in the left submandibular area measuring $2.5 \times 2.5 \mathrm{~cm}$. There were no other abnormalities in the nasopharynx, oral cavity, larynx or ears, and no palpable cervical lymph nodes. The results of the laboratory tests, including the differential white blood cell count, were all within normal limits, and no leukocytosis or anemia was noted.

Chronic sialolithiasis with sialadenitis was suspected, and the patient was administered a course of antibiotics for 2 weeks. However, the treatment did not improve her condition. An helical computed tomography (CT) scan revealed an enlarged left submandibular gland (Fig. 1A) without any clearly enlarged lymph nodes. In addition, there were heterogeneous enhancements with multiple microcysts in the bilateral parotid
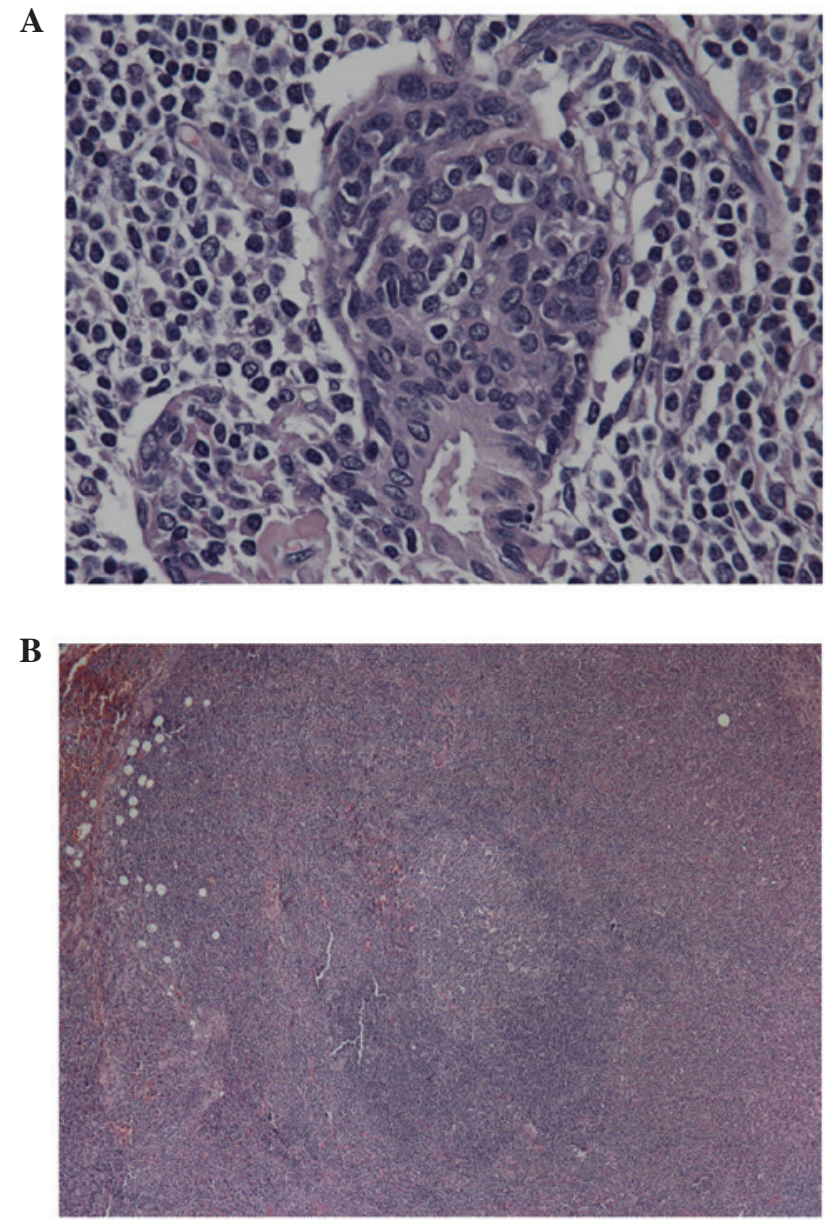

Figure 2. Microscopy and photomicrography of the submandibular gland (A) Microscopic section exhibited neoplastic and monocytoid B-cells present in the proliferative ducts (hematoxylin and eosin stain, $\mathrm{x} 400$ ). (B) Photomicrograph demonstrated atypical lymphoid cells surrounding the reactive follicular centers (hematoxylin and eosin stain, $\mathrm{x} 40$ ).

glands (Fig. 1B). Thus, the patient underwent ablation of the left submandibular gland for cosmetic reasons and to rule out the presence of a malignant growth. The size of the resected gland was $3.5 \times 2.5 \mathrm{~cm}$.

The pathology report was consistent with an extranodal low-grade marginal zone B-cell lymphoma of MALT lymphoma characterized by a lympho-epithelial lesion. Aggregates of neoplastic marginal and monocytoid B-cells were identified in the focally proliferative ducts of the salivary gland (Fig. 2A). Additionally, the majority of the normal salivary parenchyma were replaced by neoplastic lymphoid cells. There were many reactive follicular centers surrounded by an infiltration of atypical small- to medium-sized lymphoid cells (Fig. 2B; magnification, $\mathrm{x} 40$ ). At $\mathrm{x} 400$ magnification, these atypical lymphoid cells exhibited irregular nuclei with hyperchromatia, chromatin clumping, occasional prominent small nucleoli and abundant, partially pale cytoplasm.

Immunohistochemical (IHC) analysis was performed using an automated IHC stainer; Leica Bondmax (Leica Biosystems, Milton Keynes, UK). IHC against cluster of differentiation (CD)3, CD5, CD10, CD20, CD23, Cyclin-D1, B-cell lymphoma 2 (Bcl-2), $\mathrm{IgG} 4, \kappa$ and $\lambda$ light chains and in situ hybridization of Epstein-Barr virus were performed using the commercialized kits provided by Leica. The results of the IHC analysis indicated that these 

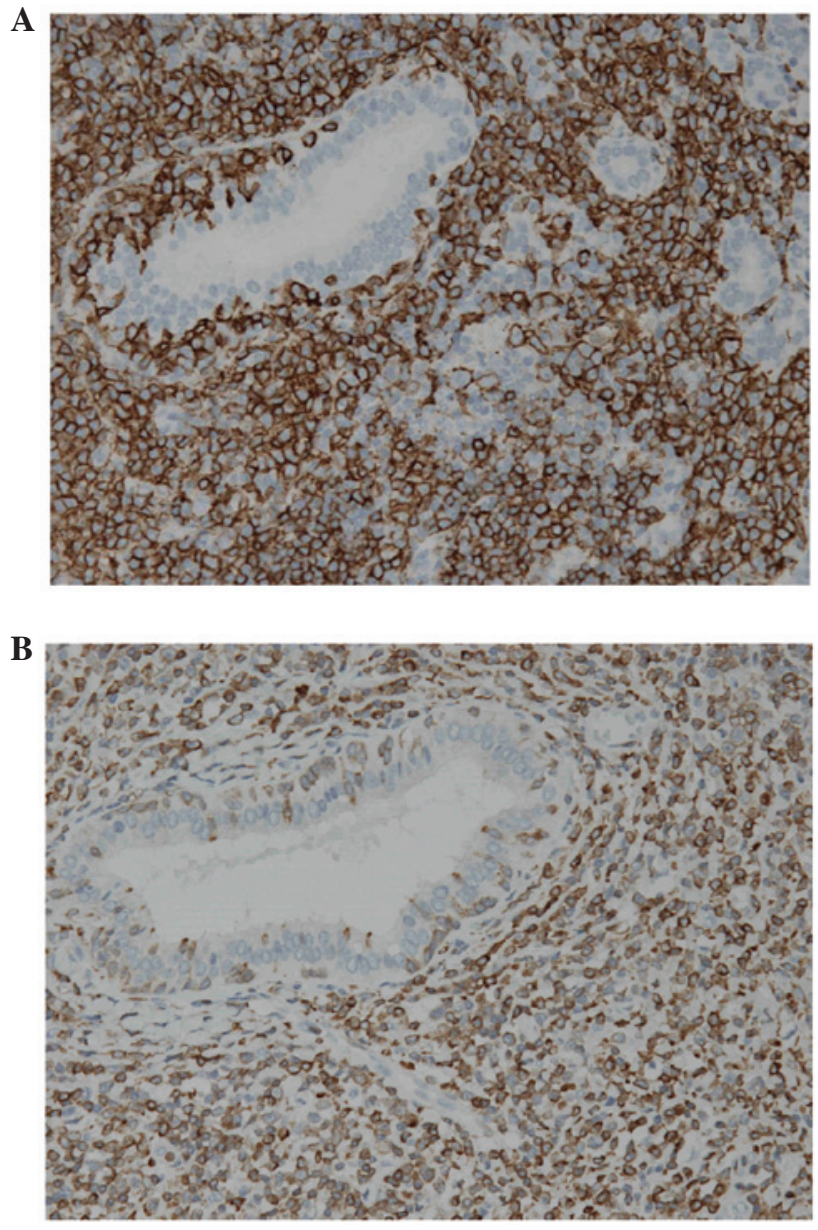

Figure 3. Immunophenotypic staining and immunohistochemical (IHC) analysis of the lesion in monocytoid cells. (A) IHC staining was positive for cluster of differentiation 20 (CD20; original magnification, x200) (B) Immunohistochemistry was positive for B-cell lymphoma 2 (Bcl-2; original magnification, $\mathrm{x} 200$ ).

monocytoid cells were positive for B-lymphocyte antigen CD20 and Bcl-2 (Fig. 3A and B), but negative for CD3, 5, 10 and 23, and for cyclin-D1 and B-cell lymphoma 1. Immunohistochemical analysis for $\lambda$ and $\kappa$ light chains demonstrated $\lambda$ chain restriction, suggesting that the monocytoid cells were monoclonal in origin. The results of Epstein-Barr virus test using in situ hybridization, and the immunoglobulin (Ig) G4 immunostaining test for the detection of IgG4-associated sialadenitis, were negative.

The patient was then referred to the Department of Hematology and Oncology (China Medical University Hospital) for further assessment and management. Additional tests were conducted, including a test for $H$. pylori infection, intestinal metaplasia and lymphoma-associated alterations in the gastrointestinal tract were examined by performing upper gastroendoscopy and colonfiberscopy. However, the results of all the tests performed were negative. Postoperative bone marrow biopsy indicated no lymphoma infiltration, while positron emission tomography/CT scan demonstrated no other malignancy.

Although the patient did not complain of any oral or ocular symptoms, such as dryness or pain, she was referred to the Department of Rheumatology (China Medical University Hospital) for further evaluation. The results of the Schirmer's, Saxon and human immunodeficiency virus (HIV) tests were negative; the result for rheumatoid factor was also negative; and serum complement components C3 (86.9 mg/dl; normal range, $79-152 \mathrm{mg} / \mathrm{dl})$ and $\mathrm{C} 4$ (19.7 $\mathrm{mg} / \mathrm{dl}, 16-38 \mathrm{mg} / \mathrm{dl})$, and IgG level (1,010 mg/dl, normal range, $751-1560 \mathrm{mg} / \mathrm{dl})$, were all within normal limits. However, the antinuclear antibody test revealed a speckled appearance, while serological markers for Sjögren's syndrome A (SS-A) were strongly positive. Salivary gland scintigraphy demonstrated reduced trapping function and excretion of radio-pertechnetate in the salivary glands.

Despite the absence of clinical symptoms, Sjögren's syndrome was then considered, based on the laboratory and radiography findings. Using the Ann Arbor staging system (8), the patient was diagnosed with MALT lymphoma, stage I with extralymphatic organ involvement, with underlying SS-A. Although the patient was advised adjuvant therapy for MALT lymphoma, she chose not to, but did attend regular follow-ups for the management of her primary Sjögren's syndrome (pSS). There was no evidence of tumor recurrence over the 6-month follow-up period subsequent to surgery.

\section{Discussion}

Extranodal MALT lymphoma is an indolent B-cell non-Hodgkin lymphoma that develops in organs that normally lack lymphoid tissue, including the stomach, lungs, ocular adnexa and salivary glands (7). A local, chronic, antigen-driven stimulation triggers the development of organized lymphoid tissue (7). B-cell accumulation usually occurs in response to chronic inflammation associated with chronic infection or autoimmune diseases, including SS, systemic lupus erythematous (SLE), and Hashimoto thyroiditis (7). These disorders are considered to be associated with the development of MALT lymphoma (7), and the stomach is the most commonly affected organ, often as a result of chronic $H$. pylori infection.

MALT lymphoma of the salivary glands is rare: In a recent study by Vazquez et al (9), salivary gland MALT lymphomas incidence rate was reported as 0.086 cases $/ 100,000$ indivduals. The parotid gland was involved in $80.87 \%$ of all the cases. MALT lymphoma of the submandibular gland is less common, according to Vazquez et al (9) only $14.40 \%$ MALT lymphomas arise from the submandibular gland, with an isolated submandibular swelling associated with MALT lymphoma as the singular symptom. Reports in the literature confirm that lymphoma is rarely the initial presenting symptom in patients with SS; usually, patients with this disorder experience other clinical symptoms prior to the development of MALT lymphoma (10). Furthermore, pSS often manifests in the parotid region, and submandibular involvement is uncommon $(11,12)$. Symptoms, such as xerostomia or xerophthalmia, are induced by chronic inflammation of the secretory organs, and usually appear prior to the development of MALT lymphoma (13).

A meta-analysis report concluded that patients with SS present a higher risk of lymphoma compared with those with other autoimmune diseases, such as SLE and rheumatoid arthritis (14). A previous study has also demonstrated that the relative risk of the development of lymphoma in patients with Sjögren's syndrome is 44 times higher than in other individuals, and that clinically identifiable lymphomas develop in $\sim 5 \%$ of these patients (15). A single-center, retrospective 
study reported that patients with early-onset SS present a high incidence of lymphoma, but not of MALT lymphoma (3).

The CT imaging of the patient in the present case report demonstrates an heterogeneous appearance, with evidence of multiple microcystic changes in the major salivary glands. These findings are typically observed in sarcoidosis, benign lympho-epithelial lesions associated with HIV infection and chronic inflammatory diseases, such as SS (16,17). According to the American-European Consensus Group criteria for pSS (18), $\geq 4$ of the following 6 criteria must be present for a diagnosis of pSS: i) Oral symptoms; ii) ocular symptoms; iii) focal sialadenitis evidenced by biopsy of the minor salivary glands; iv) evidence of kerato-conjunctivitis sicca; v) presence of SS-A or -B antigen autoantibodies; and vi) changes in the major salivary glands. Although the patient did not fulfill the diagnostic criteria, her diagnosis of MALT lymphoma due to pSS was based on expert opinion, serological and imaging analyses, and the negative outcome of a series of tests aimed to investigate other possible etiologies.

The prognosis of patients with MALT lymphoma is excellent, and the 5-year survival rate is $90 \%$ (14). Nonetheless, extragastric MALT lymphomas tend to be more aggressive than those of gastric origin, and they may recur in regional or distant lymph nodes and in other organs that are prone to MALT lymphomas (19). A previous study observed no significant difference in the survival of patients with MALT lymphomas treated with localized therapies, including surgery or radiation therapy, or systemic therapies, such as chemotherapy and/or immunotherapy with rituximab (14).

Currently, there is no standard treatment for MALT lymphoma resulting from pSS (14). A retrospective study reported that surgery is not a prognostic factor for survival in patients with MALT lymphoma in $\geq 1$ salivary glands, and that radiotherapy for early-stage disease does not confer long-term remission (11). A systematic review also indicated that asymptomatic patients with localized MALT lymphomas and without bone marrow involvement or lymphadenopathy, and an international prognostic index score of $0-1$, can be treated conservatively with regular follow-ups and no therapeutic intervention (14). In addition, the pSS disease activity may be a prognostic factor for the progression of lymphoma and the requirement for intensive management (20). The patient in the current report, despite refusing post-operative adjuvant therapy, presented no signs of tumor recurrence in the 6 months of follow-up subsequent to surgery.

In conclusion, although MALT lymphoma of the salivary glands is a well described condition, these lymphomas are most often identified in the parotid gland, and a single submandibular swelling and MALT lymphoma are rare symptoms of pSS. A multidisciplinary team that includes rheumatologists and head and neck oncologists is required for managing pSS and MALT lymphoma. Although uncommon, lymphoid malignant changes should be part of the differential diagnosis in patients who present with a single submandibular gland swelling, and should be subjected to extensive investigation aimed to identify possible underlying diseases.

\section{References}

1. Fox RI: Sjogren's syndrome. Lancet 366: 321-331, 2005.
2. Kassan SS and Moutsopoulos HM: Clinical manifestations and early diagnosis of Sjogren syndrome. Arch Intern Med 164: 1275-1284, 2004

3. Ramos-Casals M, Cervera R, Font J, García-Carrasco M, Espinosa G, Reino S, Pallarés L and Ingelmo M: Young onset of primary Sjögren's syndrome: Clinical and immunological characteristics. Lupus 7: 202-206, 1998.

4. Qin B, Wang J, Yang Z, Yang M, Ma N, Huang F and Zhong R: Epidemiology of primary Sjögren's syndrome: A systematic review and meta-analysis. Ann Rheum Dis 74: 1983-1989, 2015.

5. Singh AG, Singh S and Matteson EL: Rate, risk factors and causes of mortality in patients with Sjögren's syndrome: A systematic review and meta-analysis of cohort studies. Rheumatology (Oxford) 2015. [Epub ahead of print], 2015.

6. Fazaa A, Bourcier T, Chatelus E, Sordet C, Theulin A, Sibilia J and Gottenberg JE: Classification criteria and treatment modalities in primary Sjögren's syndrome. Expert Rev Clin Immunol 10: 543-551, 2014

7. Thieblemont C, Bertoni F, Copie-Bergman C, Ferreri AJ and Ponzoni $\mathrm{M}$ : Chronic inflammation and extra-nodal marginal-zone lymphomas of MALT-type. Semin Cancer Biol 24: 33-42, 2014.

8. Lee SE, Paik JS, Cho WK, Choi BO, Lee SN, Jung SE, Park KS, Kang CS, Kim SH, Yang SW and Cho SG: Feasibility of the TNM-based staging system of ocular adnexal extranodal marginal zone lymphoma of mucosa-associated lymphoid tissue (MALT lymphoma). Am J Hematol 86: 262-266, 2011.

9. Vazquez A, Khan MN, Sanghvi S, et al: Extranodal marginal zone lymphoma of mucosa-associated lymphoid tissue of the salivary glands: a population-based study from 1994 to 2009 . Head Neck 37: 18-22, 2015.

10. Voulgarelis M, Dafni UG, Isenberg DA and Moutsopoulos HM: Malignant lymphoma in primary Sjögren's syndrome: A multicenter, retrospective, clinical study by the European Concerted Action on Sjögren's syndrome. Arthritis Rheum 42: 1765-1772, 1999.

11. Anacak Y, Miller RC, Constantinou N, Mamusa AM, Epelbaum R, Li Y, Calduch AL, Kowalczyk A, Weber DC, Kadish SP, et al: Primary mucosa- associated lymphoid tissue lymphoma of the salivary glands: A multicenter Rare Cancer Network study. Int J Radiat Oncol Biol Phys 82: 315-320, 2012.

12. Movahed R, Weiss A, Velez I and Dym H: Submandibular gland MALT lymphoma associated with Sjögren's syndrome: Case report. J Oral Maxillofac Surg 69: 2924-2929, 2011.

13. Maślińska M, Przygodzka M, Kwiatkowska B and Sikorska-Siudek K: Sjögren's syndrome: Still not fully understood disease. Rheumatol Int 35: 233-241, 2015.

14. Kassan SS, Thomas TL, Moutsopoulos HM, Hoover R, Kimberly RP, Budman DR, Costa J, Decker JL and Chused TM: Increased risk of lymphoma in sicca syndrome. Ann Intern Med 89: 888-892, 1978 .

15. Routsias JG, Goules JD, Charalampakis G, Tzima S, Papageorgiou A and Voulgarelis M: Malignant lymphoma in primary Sjögren's syndrome: An update on the pathogenesis and treatment. Semin Arthritis Rheum 43: 178-186, 2013.

16. Cho CM, Tong SL, Bhatia KS, Wong KT, Yuen HY, Lee YP and Ahuja AT: Unusual parotid gland lesions: A pictorial review. J Clin Ultrasound 41: 501-508, 2013.

17. Rastogi R, Bhargava S, Mallarajapatna GJ and Singh SK: Pictorial essay: Salivary gland imaging. Indian J Radiol Imaging 22: 325-333, 2012.

18. Shiboski SC, Shiboski CH, Criswell L, Baer A, Challacombe S, Lanfranchi H, Schiødt M, Umehara H, Vivino F, Zhao Y, et al; Sjögren's International Collaborative Clinical Alliance (SICCA) Research Groups: American College of Rheumatology classification criteria for Sjögren's syndrome: A data-driven, expert consensus approach in the Sjögren's International Collaborative Clinical Alliance cohort. Arthritis Care Res (Hoboken) 64: 475-487, 2012.

19. Wenzel C, Fiebiger W, Dieckmann K, Formanek M, Chott A and Raderer M: Extranodal marginal zone B-cell lymphoma of mucosa-associated lymphoid tissue of the head and neck area: High rate of disease recurrence following local therapy. Cancer 97: 2236-2241, 2003.

20. Pollard RP, Pijpe J, Bootsma H, Spijkervet FK, Kluin PM, Roodenburg JL, Kallenberg CG, Vissink A and van Imhoff GW: Treatment of mucosa-associated lymphoid tissue lymphoma in Sjogren's syndrome: A retrospective clinical study. J Rheumatol 38: 2198-2208, 2011. 